\title{
Differences in Antibiotic Resistance of MDR Enterobacteriaceae between Broiler and Laying Farms in Which Two mcr-1 Positive Plasmids Revealed their Risk of Resistance
}

\author{
Shuaizhou Zong \\ Zhejiang Gongshang University \\ Dingting $X u$ \\ Second Affiliated Hospital of Zhejiang University \\ Xiner Zheng \\ Zhejiang Gongshang University \\ Peng Wang \\ Zhejiang Gongshang University \\ Jianzhong Han \\ Zhejiang Gongshang University \\ Daofeng Qu ( $\nabla 125800160 @ q q . c o m$ ) \\ Zhejiang Gongshang University
}

\section{Research Article}

Keywords: Difference, ARGs, MGEs, multidrug-resistant Enterobacteriaceae, broiler and laying farms

Posted Date: January 24th, 2022

DOI: https://doi.org/10.21203/rs.3.rs-1274477/v1

License: (a) (1) This work is licensed under a Creative Commons Attribution 4.0 International License.

Read Full License 
broiler and laying farms in which two mcr-1 positive plasmids revealed their risk

4 Shuaizhou Zong ${ }^{\mathrm{a}}$, Dingting $\mathrm{Xu}^{\mathrm{b}}$, Xiner Zheng, Peng Wang ${ }^{\mathrm{a}}$, Jianzhong Han ${ }^{\mathrm{a}}$, Daofeng $5 \quad \mathrm{Qu}^{\mathrm{a}, *}$

6

$7 \quad$ aZhejiang Gongshang University, Hangzhou 310035, PR China

$8{ }^{\text {b}}$ The second affiliated hospital, school of medicine, Zhejiang university, Hangzhou

9311100 , Zhejiang, People's Republic of China

10

11 *Corresponding Author: Daofeng Qu

12 E-mail addresses: 125800160@qq.com 


\section{Abstract}

Background: Existing studies on poultry (broilers and layers) suggest that strong screening of antibiotics leads to the wide spread of antibiotic resistance genes (ARGs). However, due to the different breeding cycle, antibiotic use and breeding environment characteristics of layers and broilers, they have different antibiotic resistance risks. Until now, there are few studies on the difference in risk of antibiotic resistance between broilers and layers, which is still a gap in our knowledge.

Results: In the current study, high-throughput sequencing-based metagenomic revealed that firmicutes and bacteroidetes were the dominant bacteria in broiler and layer farms. Aph(3',')-III and ermF were the ARGs with the highest absolute abundance in broiler farms, which was higher than that of laying farms. Tet $W$ was the highest absolute abundance of ARG in laying hens, but it was still lower than broiler chickens. Three hundred fecal samples were used to isolate multidrug-resistant Enterobacteriaceae (MDRE). It was found that the detection rates of $m c r-1$, aph(3')-Ia, aadA2, bla oXA-1, bla ${ }_{C T X-M}, f o s B, q n r D$, sul1, tetA and catA1 in broiler source MDRE were significantly higher than that in laying farms $(\mathrm{P}<0.05)$. Further studies showed that a chimeric plasmid p20432-mcr which carried the novel integron In 1866 was isolated from broiler source MDRE. High frequence of conjugation $\left(10^{-1}\right.$ to $10^{-3}$ ) and wide range of hosts made p20432-mcr likely play an important role for the high detection rate of $m c r-1, \operatorname{aph}\left(3^{\prime}\right)-I a$ and $\operatorname{addA2}$ in broiler farms.

Conclusions: We comprehensively compared the prevalence of pathogen MDRE and their ARGs in the two environments and the mechanism of transmission. It is found that the $m c r-1$ positive plasmid p20432-mcr has a wide host range which may play an important part in the high detection rate of ARGs in broiler source MDRE. These findings will help to optimize disinfection and antibiotic resistant bacteria surveillance 
38 programs in poultry farms, especially in broilers.

39 Keywords: Difference, ARGs, MGEs, multidrug-resistant Enterobacteriaceae,

40 broiler and laying farms

41 


\section{Introduction}

Antibiotic resistance has been characterized as a global public health concern, which catalyzed by the large amounts of antibiotics used in the production of food-producing animals [1,2]. Every year, hundreds of tons of antibiotics used to treat diseases and growth promoters in livestock and poultry [3, 4]. This has caused the accumulation of antibiotics in farmed environment, creating the constant selective pressure for antibiotics resistance to microorganisms [4], which make poultry become the reservoirs of resistant bacteria and resistant genes [5]. Then antibiotics resistance genes (ARGs) which can be transferred to human pathogens and enter the human food chain [6]. Therefore, transmission of resistant genes and their hosts in the farm environment needs to be monitored more frequently.

China is home to the largest layer chicken industry in the world, and is the second-largest producer of broiler chickens [7,8]. These industries cause massive pollution of the environment by animal excretion [9]. The high numbers of resistant bacteria that are often isolated in feces turn farms into outposts for the spread of resistant bacteria [10]. ARGS mostly derived from the Enterobacteriaceae which have become increasingly common in human and animal infections [11]. Polymyxin is generally considered the last resort for the treatment of multidrug-resistant Enterobacteriaceae (MDREs) [12]. Although the Chinese government has banned colistin as a growth-promotor in livestock and poultry production [13]. With the wide spread of colistin resistance genes and increasing rates of MDRE [14], contamination of livestock and poultry products with these foodborne pathogens remains a major 
risk to public health and may jeopardize the treatment of serious bacterial infections [14-16].

To date, most studies on antibiotic resistance in poultry production mainly focus on antibiotic residues and specific ARGs, and there are few studies on separate comparison between broilers and layers [16]. There is a knowledge gap in our understanding of the differences in bacterial community structure, antibiotic resistome, and mobile genetic elements (MGEs) between layers and broilers environment.

In this study, high-throughput sequencing-based metagenomic was used to analysis the abundance of ARGs and their potential bacterial hosts in the microbial communities. We conducted collect 300 fresh feacal samples from farm environmental of 3 broiler farms (BFs) and 3 laying farms (LFs) in Zhejiang, China, to characterize the difference of antimicrobial resistance in MDR Enterobacteriaceae (MDRE) between broiler and laying husbandry and analyze the multidrug resistance profiles of broilers and layers. We identified the detection of antibiotic resistance genes in MDRE that are prohibited or have different antibiotic requirements in broilers and layers. In particular, polymyxins are critically important for the treatment of MDRE infections in humans. Polymyxin resistance gene $m c r-1$ was detected in both BFs and LFs. However, we analyzed two plasmids which carried $m c r-1$, and the chimeric plasmid p20432-mcr from BFs was a multidrug-resistant plasmid contains both IncHI2 type and IncN type replicons which make it have a wide range of hosts. Overall, this study provides a theoretical basis for the differential management of broiler and layer breeding systems in China and beyond. 


\section{Methods and Material}

\subsection{Study area and sample collection}

Six farms corresponding to layers and broilers were selected for comparative study, all farms were located in Jiaxing, Zhejiang Province. None of the six farms were large intensive operations but small farms with chicken houses, which is very common in the local area. Each farm has four to six individual chicken houses and each house has about 80 chickens. Twenty fecal samples were randomly collected at each chicken house by swab. 150 fecal samples in BFs and 150 fecal samples in LFs were collected respectively in September 2021. In addition, soil $(n=3)$, water $(n=3)$, and faces $(n=3)$ samples were collected from broiler farms and mixed into a composite sample. The same sampling scheme is also used in layer farms. A total of two composite samples were used for metagenomic analysis. All swab samples were transported to laboratory in normal saline within 8 hours for isolation of

\section{Enterobacteriaceae.}

\subsection{Metagenomic analysis}

Metagenomic sequencing was performed using Illumina Hiseq 4000 with the sequencing strategy of Index 250 PE (paired-end sequencing). Raw data is filtered using fastp [17] in order to correct base and quality-filtered. Low-quality reads were filtered to ensure that (1) the reads were aligned to proper adaptors or primers, (2) the reads contained $<10 \%$ unknown bases, (3) the reads contained $>50 \%$ high-quality bases [18]. Bowtie2 [19] was used to remove reads from the host genome or contamination with high similarity. On average, 9.5 GB clean reads were generated 
for each sample. ARG-OAP PROTO [20], an analysis pipeline, integrates two common resistance gene databases, including ARDB [21] and CARD [22], identifies assembly sequences based on hidden Markov model (HMM) was used to characterize the profile and quantify the relative abundance of ARGs.

\subsection{Antimicrobial Susceptibility Test}

The antimicrobial susceptibility of isolated strains were tested with the disk diffusion method which is recommended by Clinical \& Laboratory Standards Institute (CLSI) [23] on Muleller-Hinton(MH) agar and evaluated based on the size of inhibition zones and classified as resistant, intermediate, or susceptible (the breakpoints were shown in Supplementary Table1). The strains exhibiting resistance to three or more classes of antimicrobials were considered to be multi-drug resistant organisms.

\subsection{PCR amplification and sequencing of 16S rRNA.}

The isolated strains were identified by VITEK-2 Compact and 16S rRNA gene identification using the universal 16s primers $27 \mathrm{~F}$ and 1492R [24]. The system amplified by $5 \mu \mathrm{L}$ PCR was added into $1.2 \%$ agarose gel for electrophoresis, and the voltage was set at $120 \mathrm{~V}$ for $30 \mathrm{~min}$. PCR amplification consisted of denaturation at $96{ }^{\circ} \mathrm{C}$ for $5 \mathrm{~min}$ followed by denaturation at $95^{\circ} \mathrm{C}$ for $30 \mathrm{~s}$, annealing at $50{ }^{\circ} \mathrm{C}$ for $90 \mathrm{~s}$, and polymerization at $72{ }^{\circ} \mathrm{C}$ for 60 s for a total of 30 cycles, and a final extension at $72{ }^{\circ} \mathrm{C}$ for $5 \mathrm{~min}$. Western blot detection system was used to observe whether agarose gel had corresponding bands at $1500 \mathrm{bp}$ after electrophoresis. PCR product of successful amplification was used paired-end sequencing (2x150bp ) by Sangon 
Biotech (Shanghai, China).

\subsection{PCR amplification and sequencing of Antibiotic Resistance Genes}

The isolated strains were screened for nine major classes of antibiotic resistance genes which are usually used for human clinical treatment or poultry by PCR using the primers listed in Supplementary Table2. PCR amplification consisted of denaturation at $95{ }^{\circ} \mathrm{C}$ for $5 \mathrm{~min}$ followed by denaturation at $94{ }^{\circ} \mathrm{C}$ for $30 \mathrm{~s}$, annealing at their respective annealing temperature for $30 \mathrm{~s}$, and polymerization at $72{ }^{\circ} \mathrm{C}$ for 40 $\mathrm{s}$ for a total of 30 cycles, and a final extension at $72{ }^{\circ} \mathrm{C}$ for $10 \mathrm{~min}$. All the PCR products were subjected to Sanger sequencing.

\subsection{Conjugal Transfer Experiments}

To access the transferability of $m c r-1$ positive plasmids, conjugal transfer experiments were performed with rifampin resistant E.coli strain EC600 used as a recipient and each of the $m c r-1$ positive strain RB20432 and DB20D28 Isolates as a donor. The donor can grow in Brain Heart Infusion (BHI) agar plates with $2 \mu \mathrm{g} / \mathrm{ml}$ polymyxin and the recipient E.coli strain EC 600 can grow in BHI agar plates with $2.5 \mathrm{mg} / \mathrm{mL}$ rifampicin. After incubation at $37{ }^{\circ} \mathrm{C}$ for $24 \mathrm{~h}$, colonies growing on selective plates were further confirmed by antimicrobial sensitivity experiments and VITEK-2 Compact.

\subsection{Sequencing and Analysis of plasmids}

The two conjugatable plasmids of the transconjugants RB20432-EC600 and DB20D28-EC600 were sequenced by the Illumina HiSeq platforms. The two plasmids DNA was extracted from the transconjugants RB20432-EC600 and 
DB20D28-EC600 using the SanPrep Column Plasmid Mini-Preps Kit. After extraction, plasmid sequencing was performed by with a pair-end library with an average insert size of 300 bp on a Hiseq sequencer (Illumina). Quality control and removing low-quality data were performed with Trim Momatic 0.36 [25]. Gapcloser [26] was used to fill gaps. Finally, Cytoscape [27] was used to spline the sequence to obtain the final cyclized plasmids. The plasmid sequences were submitted to Rast [28], Genemarks, Glimmer, and Prodigal library for preliminary gene prediction then submitted to ISFinder、Tn Number Registry and Integrall [29] for MGEs.

\subsection{Statistical analysis}

The data of bacterial and ARGs were processed with Microsoft Excel 2016. Column was generated using Origin 2021. Heat map for resistance profile of MDRE was visualized via Heatmapper [30] (website URL: http://www.heatmapper.ca/2. Independent sample t-tests were used by SPSS21.0 in analysis of antimicrobial susceptibility and detection of resistance gene of MDRE isolates, and $\mathrm{P}<0.05$ was considered statistically significant. Running a gene alignment program in Perl language with Ubuntu 18.04 LTS1 and Inkscape 0.48.1 were used to draw gene organization diagrams.

\section{Results}

\subsection{Characterization of bacterial and ARG communities between LFs and BFs}

The top ten relative abundance of the microbial communities at the phylum levels were shown in Fig. 1. Firmicutes, Bacteroidetes were the top two in all bacteria phyla, with $55.16 \%$ and $32.58 \%$ in BFs sample, $44.72 \%$ and $41.88 \%$ in LFs sample. It 
can be inferred that firmicutes, bacteroidetes are the dominant bacteria in broiler and laying breeding environment which were potential host of ARGs. Actinobacteria, proteobacteria, synergistetes were the secondary dominant phyla.

The top ten antibiotic resistance genes in broiler and laying farms in absolute abundance were aminoglycosides, bacitracin, $\beta$-lactam, chloramphenicol, MLSBs (macrolide-lincosamide-streptomycin B), multidrug resistance genes, sulfonamides, tetracyclines, vancomycin and unclassified antibiotic resistance genes (Fig. 2). In general, ARGs of all ten classifications were detected in broilers, while MDR_transporter and $c p x R$ were not detected in laying hens. A large number of aminoglycoside and tetracycline resistance genes were enriched in broilers and layers, and their absolute abundance in broilers was higher than that in layers. In broiler samples, $\operatorname{aph}\left(3^{\prime \prime}\right)-I I I$ had the highest absolute abundance, followed by ermF, $16 \mathrm{~S}$ rRNA methylase, tet $Q$, etc. The absolute abundance of tet $W$ in laying sample was the highest, followed by aph(3'”)-III, tetQ, aadE, etc. The absolute abundance of vancomycin resistance gene $v a n G$ and $v a n S$ in laying samples was higher than that in broiler.

\subsection{Detection of multidrug resistant Enterobacteriaceae in LFs and BFs}

A total of 526 MDR Enterobacteriaceae were recovered from 300 fresh fecal samples, 272 in BFs and 254 in LFs. According to the total general detection of MDRE layers and broilers, E.coli whose percent reached 67.8\% (357/526) were the dominant bacteria, most strains, the rest were Salmonella $(8.6 \%, 45 / 526)$, Shigella (8.2\%, 43/526), Proteus (7.8\%, 41/526), Citrobacter (7.6\%, 40/526) (Fig. 3). 


\subsection{Differences in antibiotic-resistant phenotypes of MDRE in BFs and LFs}

MDRE isolated from 6 farms showed different resistance rates to 25 antibiotics

(Fig. 4). Overall, oxacillin had the highest resistance rate among the 25 antibiotics, 96\% in BFs and $92 \%$ in LFs. In addition, MDRE in BFs and LFs showed high resistance rates of more than $80 \%$ to five antibiotics which were amoxicillin, oxacillin, sulfamethoxazole, trimethoprim-sulfamethoxazole, and tetracycline. Comparison between the two farms showed that resistance rates of polymyxin $\mathrm{B}$, meropenem, oxacillin, amikacin, streptomycin, gentamicin, ciprofloxacin, sulfamethoxazole and chloramphenicol were significantly higher in BFs than in $\operatorname{LFs}(\mathrm{P}<0.05)$. Among them, polymyxin B, which has been banned for use in poultry production in recent years, has a high resistance rate in BFs, indicating that its resistance gene has been widely spread in the broiler environment

\subsection{Antibiotics resistance of Enterobacteriaceae in broilers was more serious than} that in layers.

In order to reflect the multidrug resistance profile of MDRE in the culture environment more directly, they were divided into three resistance levels of mild antibiotics resistance $(4 \mathrm{R} \sim 10 \mathrm{R})$, moderate antibiotics $(11 \mathrm{R} \sim 17 \mathrm{R})$ and severe drug resistance $(18 \mathrm{R} \sim 24 \mathrm{R})$ according to the number of antibiotics which MDRE is resistance to (Fig. 5). The proportion of moderate and severe resistance MDRE in BFs was higher than LFs, indicating that the MDR profile of Enterobacteriaceae in BFs was more serious. As the heatmaps show that the MDRE in LFs most resistant to amoxicillin, trimethoprim-sulfamethoxazole, oxacillin, tetracycline, trimethoprim, 
sulfamethoxazole, erythrocin and chloramphenicol (Fig. 6A). While the MDRE in BFs most resistant to amoxicillin, oxacillin, trimethoprim, sulfamethoxazole, erythrocin, tetracycline and cefazolin (Fig. 6B). It should be noticed that 24R MDRE in BFs and LFs were only sensitive to polymyxins, but 17R MDRE in LFs contain resistant strains of polymyxin which should be monitored more frequently. In addition, tetracycline, macrolides, sulfonamides in folic acid metabolic pathway inhibitors and amoxicillin in $\beta$-lactam were resistant, so the use of these antibiotics alone should be avoided as far as possible in poultry.

\subsection{Differences of ARGs detection between broiler and layer farms}

To investigate the prevalence of ARGs in the poultry environment, resistance genes of nine categories of antibiotics were detected by PCR analysis. The detection rates of ARGs, bla TEM ( $\beta$-lactamases), tetA (tetracycline), sull (sulfonamides), floR (chloramphenicol), fos $A 3$ (fosfomycin), $m p h(A)$ (macrolides), $m c r-1$ (polymyxins), $q n r S$ (quinolones) and $a a c\left(6^{\prime}\right)-I b-c r$ (aminoglycoside), were significant higher than other ARGs in the same category $(\mathrm{P}<0.05)$. The detection rates of each of the genes is detailed in the Supplementary Data1 and figure S1. In addition, we focused on antibiotic resistance genes that are banned in poultry production and used differently in layers and broilers for subsequent analysis. Some of these antibiotics, such as carbapenems and polymyxin, are only available for clinical use but not for use in animals. To avoid antibiotic residues in eggs, some antibiotics are banned during laying period, such as tetracycline, quinolones (danofloxacin, pefloxacin, norfloxacin, ciprofloxacin, enrofloxacin, ofloxacin), macrolides (kitasamycin), $\beta$-lactam 
(amoxicillin, ampicillin), sulfonamides (sulfachlorpyridazine sodium), polymyxin (polymyxin B).

A total of three kind of tetracycline resistance genes were detected, the detection rate of tetA all above $60 \%$ in LFs and BFs. The detection rate of TetA in BFs was significant higher than in LFs $(\mathrm{P}<0.05)$. Although TetD was detected in both LFs and BFs, there was no significant $(\mathrm{P}>0.05)$. difference (Fig. 7A). MDRE containing quinolone resistance genes $q n r A$, qnrB, $q n r D$, $q n r S$ were detected in LFs and BFs. There is a significant difference in the detection rates of $q n r D$ between BFs and LFs $(\mathrm{P}<0.05)$, and the detection rates of $q n r A, q n r B$ and $q n r D$ were all higher in BFs. While the detection rate of $q n r S$ was higher in LFs than in BFs (Fig. 7B). Among the macrolide resistance genes, the detection rate of $m p h A$ was the highest in broiler and layer farms, both exceeding 60\% (Fig. 7C). The detection rates from high to low were $m p h(A), m p h(E), m p h(B), m p h(D)$ erm $A$ and other macrolide resistance genes were not detected. The detection rate of $m p h(B)$ in BFs was significantly higher $(\mathrm{P}<0.05)$. Only three $\beta$-lactam resistance genes were identified: bla TEM, bla $a_{\text {OXA-1 }}$ and bla CTX-M $_{\text {. }}$ Except that bla $_{T E M}$ had high detection rates in both broiler and layer farms, the detection rates of bla $a_{T E M}$ and bla $a_{O A-1}$ in broiler farms were significantly higher than those in LFs $(\mathrm{P}<0.05)$ (Fig. 7D). The sulfonamides resistance genes only sull and folpl were amplified in MDRE. The total detection rate of sull was the higher than folpl in BFs and LFs (Fig. 7E). And the detection rate of sull in BFs was significantly higher than that in LFs $(\mathrm{P}<0.01)$. The detection rate of polymyxin resistance gene in broiler and layer farms was not high, and mcr-1 was detected in both farms and was 
significantly higher in BFs than in LFs $(\mathrm{P}<0.01)$ (Fig. 7F). However, $m c r-2$ was only detected in $\mathrm{BFs}$, and the detection rate was $0.32 \%$.

3.5 The mcr-1 positive plasmids p20432-mcr and p2021-mcr are conjugative.

The transconjugants isolated by mating RB20432 and DB20D28 strains with EC600 were resistant to rifampicin and polymyxin antibiotics, possibly indicating plasmids transfer. Experiments showed that polymyxin resistance could be successfully transferred from RB20432 and DB20DB to EC600 at a frequence of $10^{-1}$ to $10^{-3}$ (p20432-mcr) and $10^{-2}$ to $10^{-3}$ (p2021-mcr) cell per recipient cell by conjugation. As can be seen from the table (Table 1), the minimum inhibitory concentrations for polymyxin B for the transconjugant increasing 16-fold compared with the untransformed control. In addition, the minimum inhibitory concentration for ampicillin (32-fold) and amoxicillin (32-fold), tetracycline (32-fold), gentamicin (8-fold, only RB20432) and sulfamethoxazole (16-fold) were increasing to different degree, suggesting that resistance genes of these antibiotics also exist in p20432-mcr and p2021-mcr and be transferred through conjugation.

3.6 Characterization of multi-Drug plasmids p20432-mcr and p2021-mcr

Two mcr-1 positive multidrug-resistant plasmids p20432-mcr and p2021-mcr were found in the polymyxin resistant MDRE of fecal samples of layers and broilers, respectively. As shown in Fig. 8, the total length of plasmid 20432-mcr was 228.7kb, contained 275 ORFs in total. And it has a complex region, which has two replicons (IncHI2 and IncN), and the backbone was interrupted by accessory modules. The content of GC was $47.3 \%$, and the plasmid contained four accessory modules which 
were MDR-1 region, MDR-2 region, In1866-related region and metal resistant region. The single replicon IncN plasmid R46 (GenBank: AY046276.1) was chosen as the reference plasmid which had similar repA, intI1 and resistance genes.

The IncI2-type plasmid p2021-mcr was extracted from E. Coli strain DB20D28, with a total length of $60.2 \mathrm{~kb}$, the content was $42.3 \%$, and a total of 78 ORFs in total (Fig. 9A). Its structure was relatively simple which the whole plasmid consisted of a backbone region and a single mcr-1-pap2. mcr-1 was the only ARG in p2021-mcr. The conjugation transfer region accounted for a large proportion of the plasmid, including flagellum formation gene pilP and conjugation transfer regulation gene virB9. Among them, the Shufflon DNA-specific recombinase secreted by $r c i$ gene in this structure is the most important member of the recombinant system of IncI plasmid, which obtained exogenous DNA through specific recombination. While there were no MGEs such as IS, Tn and integron in p2021-mcr, and mcr-1 is the only ARG. IncI2 plasmids have a high overall structural similarity, while most of difference is caused by gene-specific recombination upstream and downstream of $r c i$ [31]. Linear comparison between p2021-mcr and reference plasmid pAH62-1 (Genbank: CP055260.1) (Fig. 9B) showed that mcr-1-pap2 structure also existed in the upstream of $r c i$ and the main difference was that partial sequence inversion occurred in the downstream of this gene due to recombination (Fig. 9C).

3.7 Analysis of Multi-Drug Resistant Region in p20432-mcr

The MDR-1 region of p20432-mcr is composed of MGEs and ARGs in order $\triangle I S 2$-merR-terC- $\triangle I S 2$-aph(3)-Ia-IS186B-mcr-1-PAP2-1-sul- $\triangle T n 2 . \quad m c r-1 \quad$ was 
followed by a PAP2 family of proteins, and mcr-1-pap2 has been reported in many plasmids, most of which are related to ISAp11. But in p20432-mcr, ISApl1 has been replaced by IS186B. IS 186B-mcr-1-PAP2 has no similar sequence in Genebank, but IS $186 B$ has the ability to transpose mcr-1 into chromosomes [32], which promote the vertical transmission of polymyxin resistance genes (Fig 10A). The multi-drug resistance region MDR-2 of the following mobile element in sequence IS4321, IS6100, IS26, TnAsl. IS26-mph(A)-mrx-mphR(A)-IS6100 is a classic antibiotic resistance locus which contains a macrolide resistance gene $m p h(A)$ and a regulator $\operatorname{mphR}(A)$. A new type 1 integron was found and the number In 1866 was assigned by Integrall database. The ARGs in gene cassette of In 1866 contained $d f r A 12$ (aminoglycoside) and the 3 prime-CS region contained sul3 (sulfanilamide) (Fig. and there was a long period that antibiotics(e.g. C, TET, AMP,TMP) were used to treat

325 bacterial infections or as antimicrobial growth promoters [33, 34]. As a result, 326 selection for resistant bacteria and has promoted gene mutation, recombination, and horizontal gene transfer events in poultry farming environments which can lead to the 
enrichment of multidrug resistant bacteria. China is the largest producer and consumer of eggs in the world, and second only to the United States in chicken production [35]. The enormous and still increasing demand for poultry products in China has meant that there is plenty of room to expand production of layers and broilers [36]. Identification of the phenotype and genotype of MDRE isolated from BFs and LFs indicated that different treatment methods and culture-cycle could cause the development of antimicrobial resistance in culture environment, but lead to the spread of different ARGs. Few studies have examined differences among broilers and layers, which have only focused on antimicrobial residues or in dividual resistance genes. Our understanding of the differences in antibiotic resistance profiles between broilers and layers and how they spread is still limited. Therefore, it is necessary to make effective tactics for monitor antibiotic resistance in BFs and LFs, and the monitoring focus should be different [37].

In this study, a high prevalence of MDR Enterobacteriaceae and high resistance-gene diversity has been demonstrated in poultry environment. $3 \mathrm{BFs}$ and 3 LFs were selected in Jiaxing, Zhejiang Province. 526 MDRE were isolated, of which Escherichia was the most MDR bacteria, followed by Proteus, Shigella, Salmonella and Citrobacter. There was no significant $(\mathrm{P}>0.05)$ difference in MDRE composition between laying hens and broiler farms, indicating that they are important reservoirs of ARGs in poultry farming environment. By identifying the antibiotic resistance phenotype of MDRE, the results showed that tetracycline, sulfonamides, amoxicillin and macrolides antibiotics all had high resistance rates in both types of farms. Among 
them, the resistance rates of oxacillin, amoxicillin, trimethoprim, tetracycline and

351 sulfamethoxazole used in this study have reached more than $90 \%$, while the resistance

352 rates of polymyxin $\mathrm{B}$ and meropenem are lower than $10 \%$. Tetracycline-

353 erythromycin-trimethoprim-sulfamethoxazole-sulfamethoxazole-trimethoprim-benzac

354 illin-amoxicillin are common antibiotic resistance spectrum of MDRE in this study.

355 The comparison of MDRE in BFs and LFs shows that the risk of MDRE in BFs are

356 higher than those from LFs, which is mainly reflected in the following two aspects: (1)

357 multidrug resistance rates were higher in BFs samples. (2) in terms of meropenem,

358 amoxicillin, streptomycin, gentamicin, ciprofloxacin, trimethoprim-sulfamethoxazole,

359 enrofloxacin, ofloxacin and polymyxin $\mathrm{B}$, the resistance rates of MDRE isolated from

360 BFs were higher. This result may be caused by: (1) short breeding period and weak

361 resistance of broilers; (2) compared with the cage mode of layers, free-range broilers

362 had a wider range of activities and were more susceptible to MDR infection; (3) some

363 antibiotics are forbidden in laying hens breeding or laying period, but not as strictly in

364 broilers. There were also significant differences in the detection rates of some ARGs

365 between broiler and layer farms. The detection rate of aadA2, catA1, fos $B, \operatorname{mph}(B)$,

$366 q n r B, b l a_{o x a-1}, b l a_{C T X-M}$ and tetB in BFs was significantly higher than that of LFs

$367(\mathrm{P}<0.05)$. The difference of ARGs was consistent with that of antibiotic resistance

368 phenotype, indicating that the difference was largely caused by the difference of

369 transmission of ARGs.

370 Since the plasmid carrying mor-1 was first reported in China in 2015, colistin

resistance has spread rapidly around the world by now, and the resulting development 
of Enterobacteriaceae from extensive drug resistance to pan-drug resistance is inevitable and will ultimately become global [38, 39]. A study based on the prevalence of $m c r-1$ in the human intestine showed that a decline in colistin-resistant bacteria isolated from both animals and humans decreased significantly after the implementation of the colistin prohibition policy in China in 2017 [40, 41]. However, this study found that there are still transmission risks in the breeding environment. Two $m c r-1$ positive plasmids were found to have high in-vitro transfer rates between Escherichia coli which is key human pathogen in broilers and layers respectively. In particular, p20432-mcr, found in broiler MDRE, carries a novel integron $\operatorname{In} 1866$ and multiple drug resistance genes, which is a worrying event. The plasmid p20432-mcr isolated from broiler source E.coli RB20432 belongs to a complex chimeric plasmid, which contains both IncHI2 type and IncN type replicons. The structure of multiple replicons is conducive to increasing the host range of this plasmid, which likely to make $m c r-1$ spread more widely [42]. The cytoskeleton region contains the key genes of IncHI2 type plasmid, and some fragments of IncN type plasmid were also obtained with plasmid recombination. In addition to polymyxin resistance genes, there are antibiotic resistance genes of chloramphenicol, aminoglycoside and metal resistance genes on plasmids, which make the plasmid transfer under a variety of selective pressures.

\section{Conclusion}

We conducted a comprehensive analysis of MDRE from typical broiler and laying farms in Zhejiang Province, China, and the results showed that antibiotic 
394 prohibition had a positive effect on reducing resistance genomes in the breeding 395 environment. The detection rate of some antibiotic resistance genes that were banned 396 in layers but not suppressed in broilers was significantlyincreased in broiler source 397 MDRE $(\mathrm{P}<0.05)$. However, our study shows that although certain antibiotics such as 398 polymyxin have been banned in poultry farming, the polymyxin resistance gene $\mathrm{mcr}-1$ 399 persists in the environment for a long time due to the widespread spread of mobile 400 elements such as plasmids. The analysis results of the multidrug-resistant plasmid 401 P20432-MCR showed that the multi-replicon structure enabled it to transmit $m c r-1$ 402 and other antibiotic resistant genes to different hosts, resulting in a higher detection 403 rate of $m c r-1, \operatorname{aph}\left(3^{\prime}\right)-I a$ and $a a d A 2$ in broiler farms. Therefore, it is necessary to 404 implement and improve cleaning and disinfection procedures in broiler farms, and to 405 detect resistant bacteria from broilers more frequently to prevent further transmission 406 to humans through the poultry production chain. 


\section{Abbreviations}

BFs: Brolier Farms; LFs: Laying Farms; ARGs: Antibiotic Resistance Genes; MGEs: Mobile Genetic Elements; MDR: Multidrug-Resistance; MDRE: Multidrug-Resistant Enterobacteriaceae; IS: Insertion Sequence; Tn: transposon; AMX: Amoxicillin; CZ: Cefazolin; CAZ: Ceftazidime; FOX:Cefoxitin; IPM: Imipenem; MEM: Meropenem; AT: Aztreonam; OX: Oxacillin; AK: Amikacin; KAN: Kanamycin; S:Streptomycin; GM: Gentamicin; CIP: Ciprofloxacin; ENR: Enrofloxacin; OFX: Ofloxacin; TMP: Trimethoprim; SMZ: Sulfamethoxazole; SXT: Trimethoprim-Sulfamethoxazole; C: Chloramphenicol; FOS: Fosfomycin; E: Erythromycin; AZM: Azithromycin; PB: Polymyxin B; PB: Tetracycline; MI: Minocycline.

\section{Acknowledgements}

The authors are grateful to the anonymous reviewers for their insightful suggestions and comments.

\section{Funding}

This work was supported in part by the National Natural Science Foundation of China (no. 32172188)

\section{Availability of data and materials}

All metagenomic files have been uploaded to NCBI and are under review. Once approved by NCBI, we will upload the metagenomic data number immediately.

\section{Authors' contributions}

ZSZ analyzed the data and wrote the manuscript.; XDT, ZXE analyzed the data and prepared figures. WP collected samples and helped with software. HJZ and QDF conceived the study and revised the original manuscript. All authors edited the manuscript and approved the final draft.

\section{Ethics approval and consent to participate}

There is no ethics involved in this experiment.

\section{Consent for publication}


434 Not applicable.

\section{Competing interests}

436 The authors declare that they have no competing interests.

\section{Authors' information}

$438{ }^{a}$ School of Food Science and Biotechnology, Zhejiang Gongshang University, No.18

439 Xuezheng Street, Qiantang New District, Hangzhou, Zhejiang People's Republic of

440 China. ${ }^{\text {b}}$ The second affiliated hospital, school of medicine, No. 86 Jiefang Street,

441 Shangcheng District, Hangzhou, Zhejiang, People’ s Republic of China. 


\section{References}

1. Kock R, Daniels-Haardt I, Becker K, Mellmann A, Friedrich AW, Mevius D, Schwarz S, Jurke A: Carbapenem-resistant Enterobacteriaceae in wildlife, food-producing, and companion animals: a systematic review. Clinical Microbiology \& Infection 2018:S1198743X18303392.

2. Winglee K, Howard AG, Sha W, Gharaibeh RZ, Liu J, Jin D, Fodor AA, Gordon-Larsen P: Recent urbanization in China is correlated with a Westernized microbiome encoding increased virulence and antibiotic resistance genes. Microbiome 2017, 5(1):121.

3. Xiong W, Wang Y, Sun Y, Ma L, Zeng Q, Jiang X, Li A, Zeng Z, Zhang T: Antibiotic-mediated changes in the fecal microbiome of broiler chickens define the incidence of antibiotic resistance genes. Microbiome 2018, 6(1):34.

4. Udikovic-Kolic N, Wichmann F, Broderick NA, Handelsman J: Bloom of resident antibiotic-resistant bacteria in soil following manure fertilization. Proc Natl Acad Sci U S A 2014, 111(42):15202-15207.

5. Mceachran AD, Blackwell BR, Hanson JD, Wooten KJ, Smith PN: Antibiotics, Bacteria, and Antibiotic Resistance Genes: Aerial Transport from Cattle Feed Yards via Particulate Matter. Environmental Health Perspectives 2015, 123(4):337-343.

6. Su JQ, Wei B, Ou-Yang WY, Huang FY, Zhao Y, Xu HJ, Zhu YG: Antibiotic Resistome and Its Association with Bacterial Communities during Sewage Sludge Composting. Environ Sci Technol 2015, 49(12):7356-7363.

7. Gaze WH, Krone SM, Larsson D, Li XZ, Zhu YG: Influence of Humans on Evolution and Mobilization of Environmental Antibiotic Resistome. Emerging Infectious Diseases 2013, 19(7).

8. Food Outlook - Biannual Report on Global Food Markets [http://www.fao.org/giews/]

9. Wei S, Gutek A, Lilburn M, Yu Z: Abundance of pathogens in the gut and litter of broiler chickens as affected by bacitracin and litter management. Vet Microbiol 2013, 166(3-4):595-601.

10. Faldynova M, Vídeňská $P$, Havlickova $H$, Sisak $F$, Juřicová $H$, Babak V, Steinhauser L, Rychlik I: Prevalence of antibiotic resistance genes in faecal samples from cattle, pigs and poultry. Veterinární Medicína 2013, 58(6):298-304.

11. Falagas ME, Karageorgopoulos DE, Nordmann P: Therapeutic options for infections with Enterobacteriaceae producing carbapenem-hydrolyzing enzymes. Future Microbiology 2011, 6(6):653-666.

12. Wang Y, Zhang R, Li J, Wu Z, Yin W, Schwarz S, Tyrrell JM, Zheng Y, Wang $\mathrm{S}$, Shen $\mathrm{Z}$ : Comprehensive resistome analysis reveals the prevalence of NDM and MCR-1 in Chinese poultry production. Nature Microbiology 2017, 2(02):16260. 
13. Shi X, Li Y, Yang Y, Shen Z, Wa Ng S: High prevalence and persistence of carbapenem and colistin resistance in livestock farm environments in China. Journal of Hazardous Materials 2020, 406(Supp1 4):124298.

14. Buelow E, González TB, Fuentes S, De S, Lahti L, Bayjanov JR, Majoor E, Braat JC, Mourik MV, Oostdijk E: Comparative gut microbiota and resistome profiling of intensive care patients receiving selective digestive tract decontamination and healthy subjects. Microbiome 2017, 5(1):88.

15. Belluco S, Barco L, Roccato A, Ricci A: Escherichia coli and Enterobacteriaceae counts on poultry carcasses along the slaughterline: $A$ systematic review and meta-analysis. Food Control 2016.

16. Zhai $\mathrm{R}, \mathrm{Fu} \mathrm{B}$, Shi $\mathrm{X}$, Sun $\mathrm{C}, \mathrm{Wu} \mathrm{C}$ : Contaminated in-house environment contributes to the persistence and transmission of NDM-producing bacteria in a Chinese poultry farm. Environment International 2020, 139:105715.

17. Chen S, Zhou Y, Chen Y, Jia G: fastp: an ultra-fast all-in-one FASTQ preprocessor. Bioinformatics 2018, 34(17):i884-i890.

18. Single-cell RNA-Seq profiling of human preimplantation embryos and embryonic stem cells. Nature Structural \& Molecular Biology 2013, 20(9): 1131.

19. Langmead B, Salzberg SL: Langmead B, Salzberg SL.. Fast gapped-read alignment with Bowtie 2. Nat Methods 9: 357-359. Nature Methods 2012, 9(4):357-359.

20. Yin, Xiaole, Jiang, Xiao-Tao, Chai, Benli, Li, Liguan, Yang, Ying: ARGs-OAP v2.0 with an expanded SARG database and Hidden Markov Models for enhancement characterization and quantification of antibiotic resistance genes in environmental metagenomes. Bioinformatics 2018.

21. Bo L, Mihai P: ARDB-Antibiotic Resistance Genes Database. Nucleic Acids Res 2009, 37(Database issue):D443-D447.

22. Alcock BP, Raphenya AR, Lau T, Tsang KK, Mc Arthur AG: CARD 2020: antibiotic resistome surveillance with the comprehensive antibiotic resistance database. Nucleic Acids Res 2019, 48(D1).

23. CLSI, Institute) LS: Performance Standards for Antimicrobial Susceptibility Testing: Twenty-Four Informational Supplement. CLSI document M100-S24. 2014.

24. Stackebrandt E, Goodfellow M: Nucleic acid techniques in bacterial systematics. 1991.

25. Bolger AM, Marc L, Bjoern U: Trimmomatic: a flexible trimmer for Illumina sequence data. Bioinformatics 2014(15):2114-2120.

26. $\mathrm{N}$ Ed Erbragt $\mathrm{AJ}$ : On the middle ground between open source and commercial software - the case of the Newbler program. Genome Biology 2014, 15(4):1-2.

27. Smoot, Michael E, Ono, Keiichiro, Ruscheinski, Johannes, Wang, Peng-Liang, Ideker, Trey: Cytoscape 2.8: new features for data integration and network visualization. Bioinformatics 2011. 
28. Aziz RK, Bartels D, Best AA, Dejongh M, Disz T, Edwards RA, Formsma K, Gerdes S, Glass EM, Kubal M: The RAST Server: Rapid Annotations using Subsystems Technology. BMC Genomics 2008, 9(1):75-75.

29. Alexandra Mourasup/sup, Mário Soaressup/sup, Carolina Pereirasup/sup, Nuno Leitosup/sup, Isabel Henriquessup/sup, Correiasup/sup A: INTEGRALL: a database and search engine for integrons, integrases and gene cassettes. Bioinformatics 2009, 25(8):1096-1098.

30. Sasha B, David A, Ana M, Liang Y, Grant JR, Adam M, Wishart DS: Heatmapper: web-enabled heat mapping for all. Nucleic Acids Res 2016(W1):W147-W153.

31. Gyohda A, Furuya N, Kogure N, Komano T: Sequence-specific and Non-specific Binding of the Rci Protein to the Asymmetric Recombination Sites of the R64 Shufflon. Journal of Molecular Biology 2002, 318(4):975-983.

32. Saisree L, Reddy M, Gowrishankar J: IS186 Insertion at a Hot Spot in the Ion Promoter as a Basis for Lon Protease Deficiency of Escherichia coli B: Identification of a Consensus Target Sequence for IS186 Transposition. $J$ Bacteriol 2001, 183(23):6943.

33. Niu J-L, Peng J-J, Ming Y-Y, Ma Q-C, Liu W-C, Ma Y: Identification of drug resistance genes and drug resistance analysis of Salmonella in the duck farm environment of Zhanjiang, China. Environ Sci Pollut Res Int 2020, 27(20):24999-25008.

34. National Bureau of statistics of China. [http://www.stats.gov.cn/english/]

35. Van Boeckel TP, Brower C, Gilbert M, Grenfell BT, Levin SA, Robinson TP, Teillant A, Laxminarayan $\mathrm{R}$ : Global trends in antimicrobial use in food animals. Proc Natl Acad Sci U S A 2015, 112(18):5649-5654.

36. Sahoo KC, Tamhankar AJ, Johansson E, Lundborg CS: Antibiotic use, resistance development and environmental factors: A qualitative study among healthcare professionals in Orissa, India. BMC Public Health 2010, 10(1):629.

37. Kumarasamy KK, Toleman MA, Walsh TR, Bagaria J, Woodford N: Emergence of a new antibiotic resistance mechanism in India, Pakistan, and the UK: a molecular, biological, and epidemiological study. Lancet Infectious Diseases 2010, 10(9):578-579.

38. Liu: Emergence of plasmid-mediated colistin resistance mechanism MCR-1 in animals and human beings in China: A microbiological and molecular biological study. The Lancet 2015, 16(2):161-168.

39. Cong S, Llzma D, Yyab D, Pydef G, Pdlp H, Ns I, Fm J, k MAE-GE-SAPad, Sfa D, Psh C: Dynamics of mer-1 prevalence and mcr-1 -positive Escherichia coli after the cessation of colistin use as a feed additive for animals in China: a prospective cross-sectional and whole genome sequencing-based molecular epidemiological study. The Lancet Microbe 2020, 1(1). 
571 40. Pyw A, Cxm A, Prz C, Pyc B, Ys D, Pfhe F, Dl A, Jl C, Yan G, Xi XA: Changes in colistin resistance and mcr-1 abundance in Escherichia coli of animal and human origins following the ban of colistin-positive additives in China: an epidemiological comparative study. 2020.

41. Pilla, Giulia, Tang, Christoph, M.: Going around in circles: virulence plasmids in enteric pathogens. Nature Reviews Microbiology 2018. 
579 Figure 1 Top ten relative abundance of taxonomic composition at the phylum level in

580 broiler and laying farm samples.

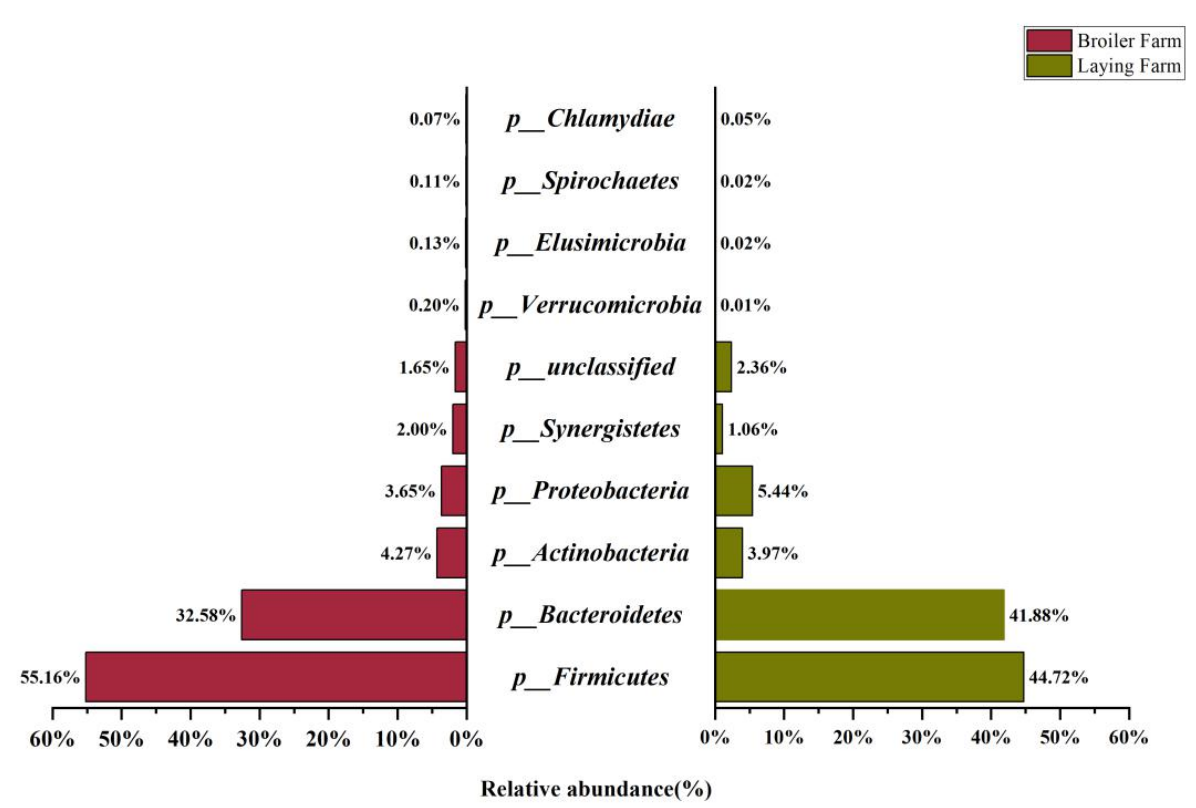

581 Figure 2 The absolute abundance of top ten antibiotic resistance gene classification

582 and their subtypes
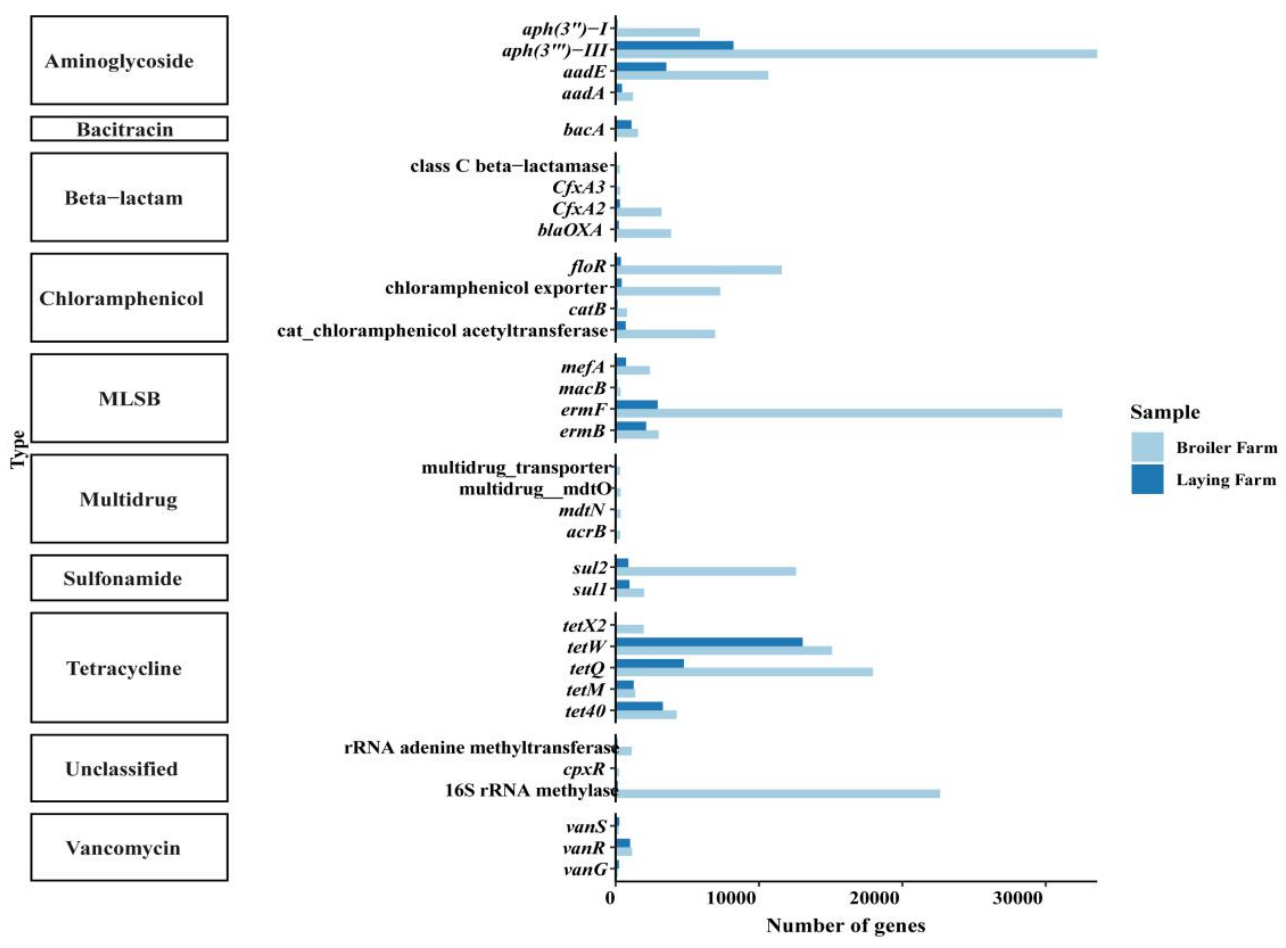


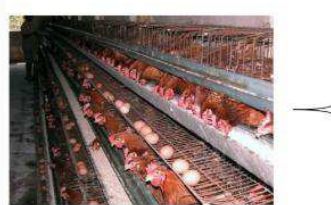

Layer Farms
Layer Farm A:

50 samples of faecal

Layer Farm B:

50 samples of faecal

Layer Farm C:

50 samples of faecal

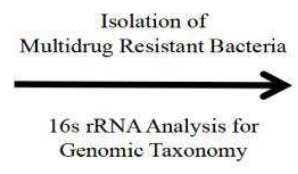

196 Escherichia

19 Salmonella

18 Shigella

25 Proteus

14 Citrobacter

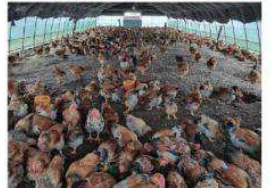

Broiler Farms

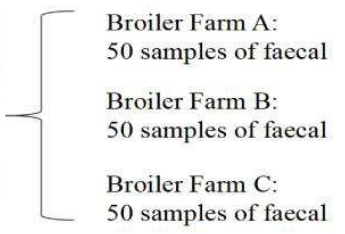
50 samples of faecal Broiler Farm B :

Broiler Farm C: 50 samples of faecal

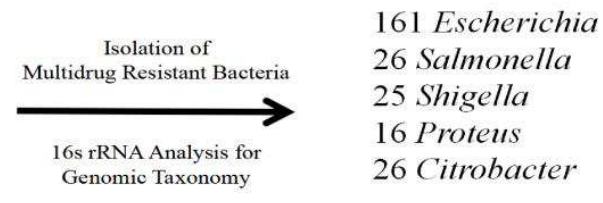

26 Salmonella

25 Shigella

26 Citrobacter

Figure 4 Resistance rates of MDRE isolated from broilers and layers to 25 antibiotics.

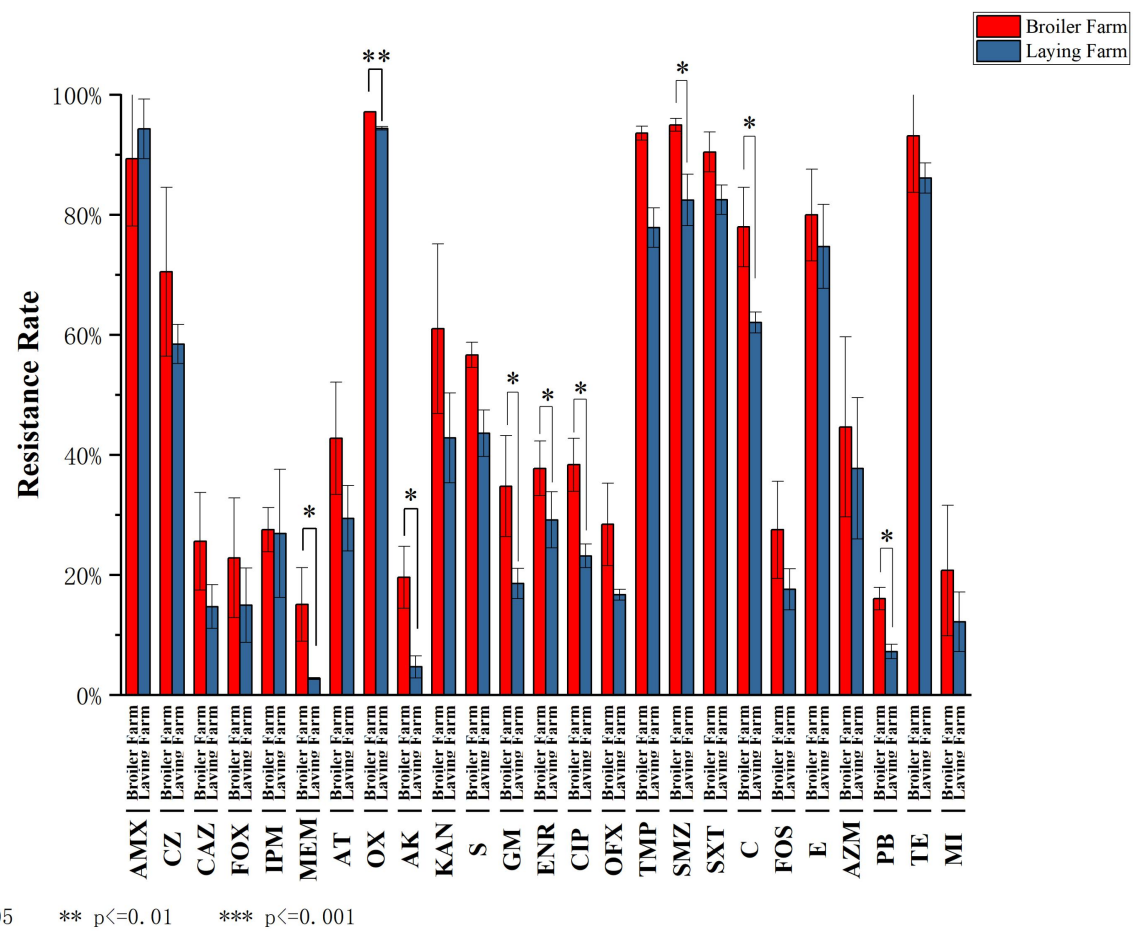

586 Figure 5 Proportion of resistant bacteria in different drug resistance situations in broiler farms and laying farms. 


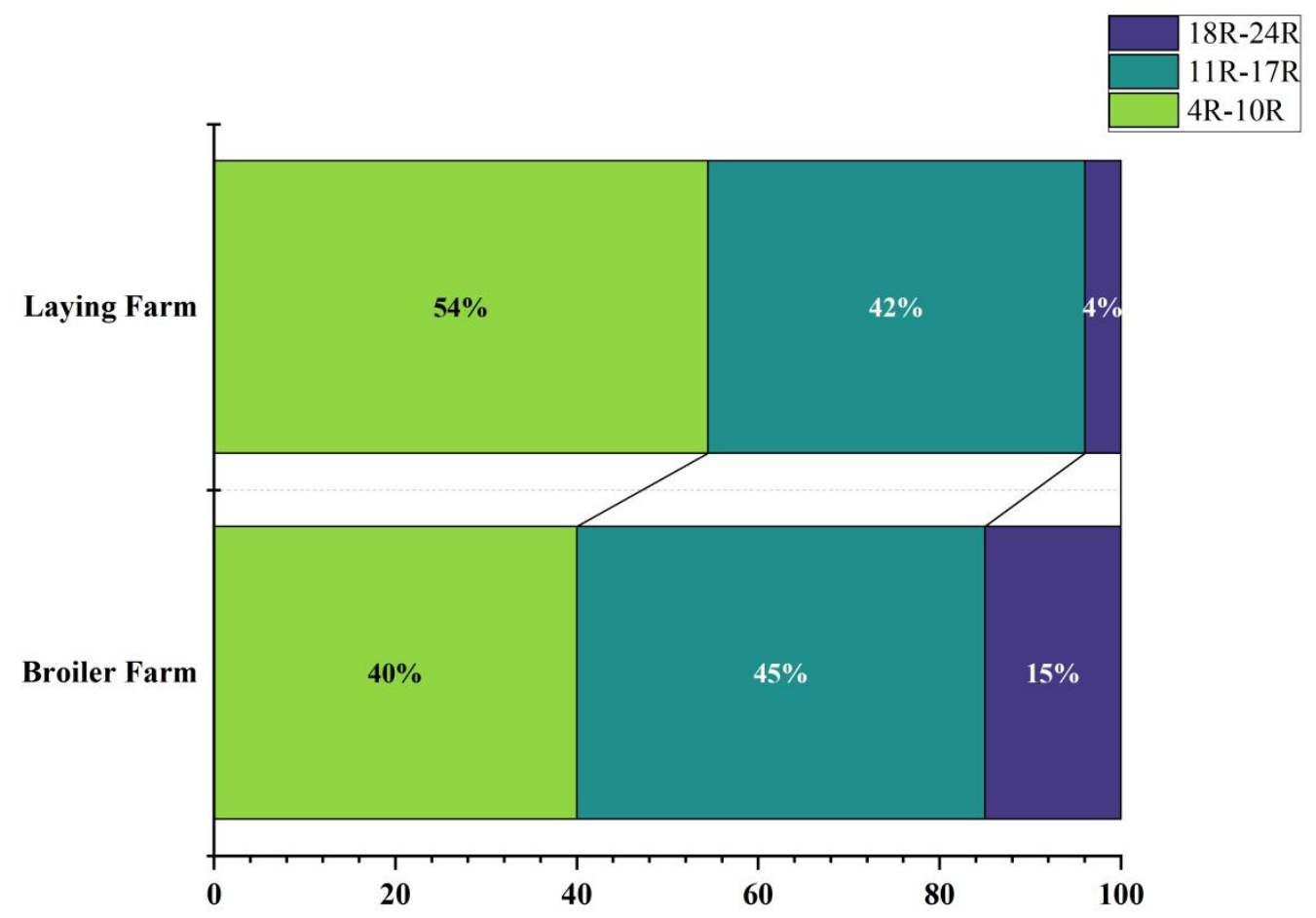

588 Figure 6 (A) Heat map of multiple drug resistance of resistant strains in broiler farms.

5896 (B) Heat map of multiple drug resistance of resistant strains in laying farms. The

590 right column shows the number of antibiotics which were resisted by MDRE.

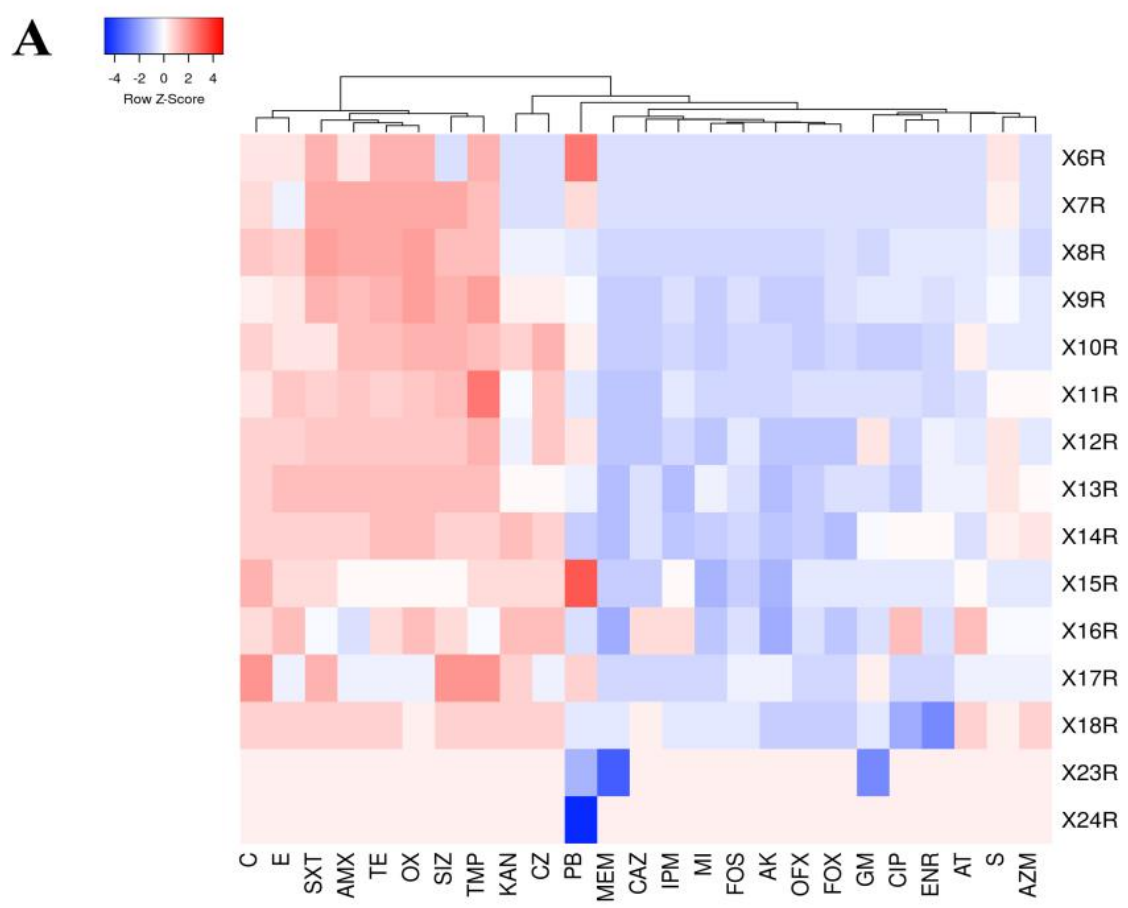




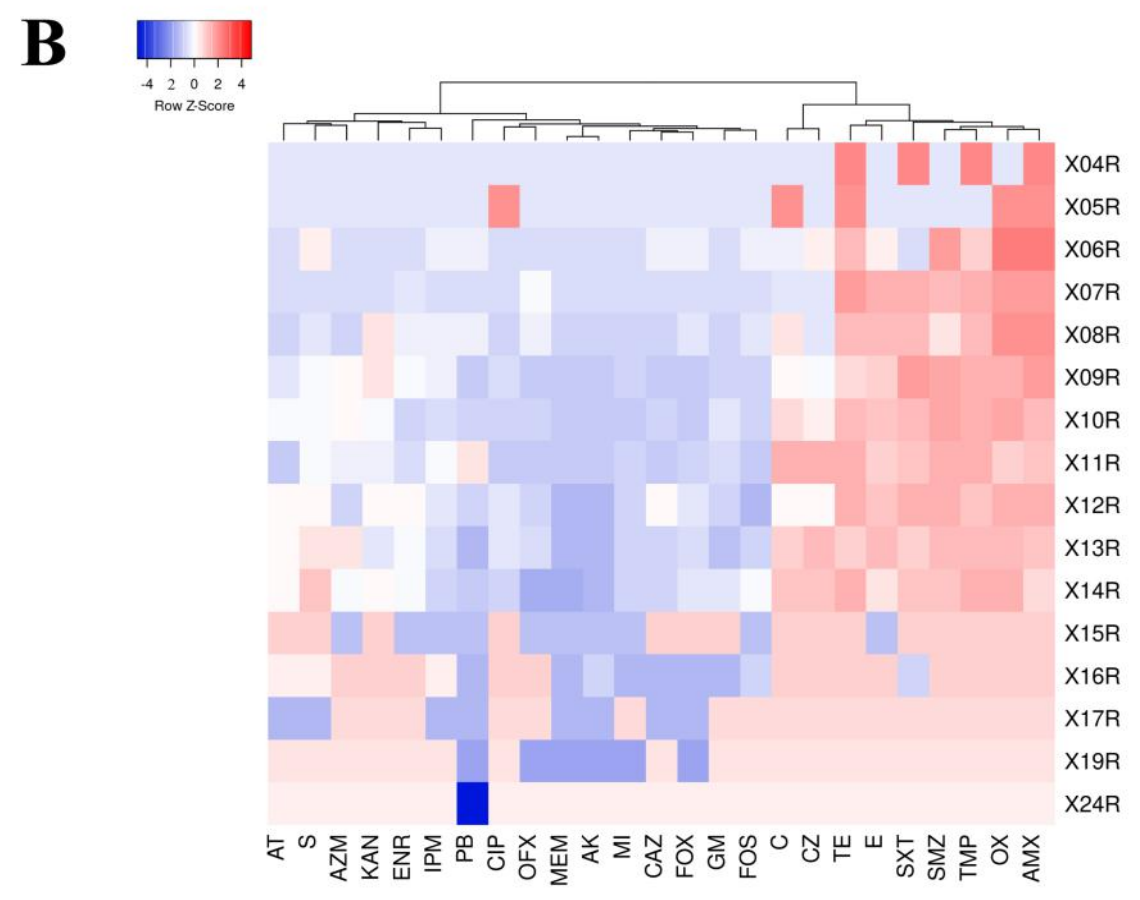

591 Figure 7 The detection rate of antibiotic resistance genes which are banned in layers 592 or both. (A) The detection rate of tetracyclines resistance genes. (B) The detection rate 593 of quinolones resistance genes. (C) The detection rate of macrolides resistance genes. 594 (D) The detection rate of $\beta$-lactam resistance genes. (E) The detection rate of 595 sulfonamide antibiotics resistance genes. (F) The detection rate of polymyxins 596 resistance genes.

A

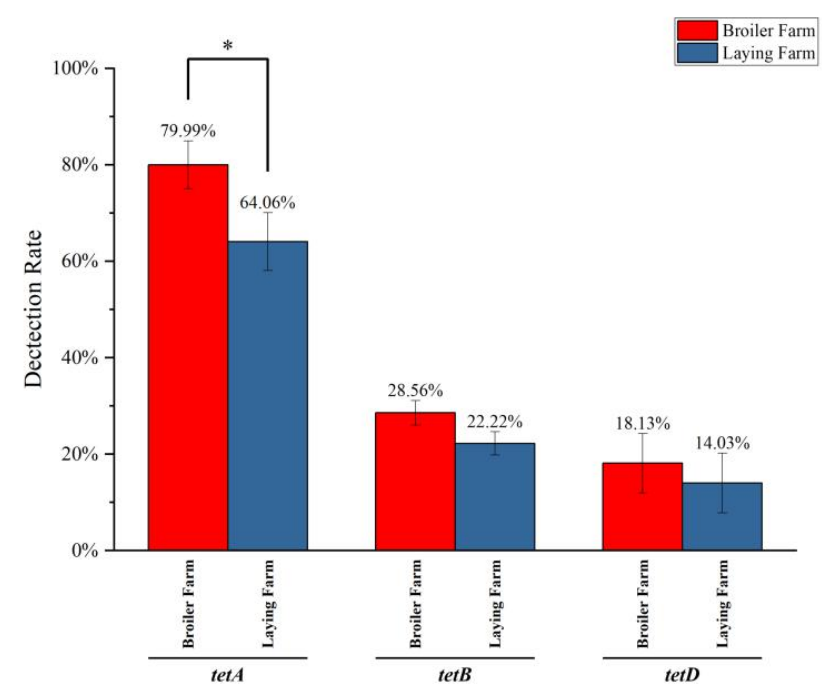


B

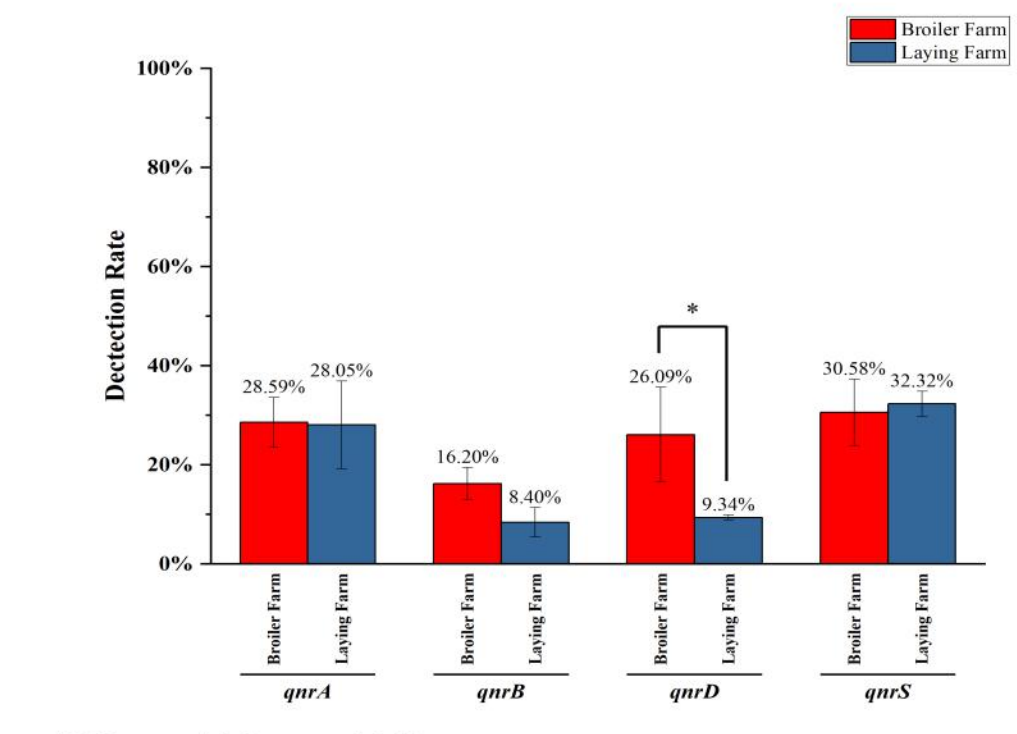

$* \mathrm{p}<=0.05 \quad * * \mathrm{p}<=0.01 \quad * * * \mathrm{p}<=0.001$

C

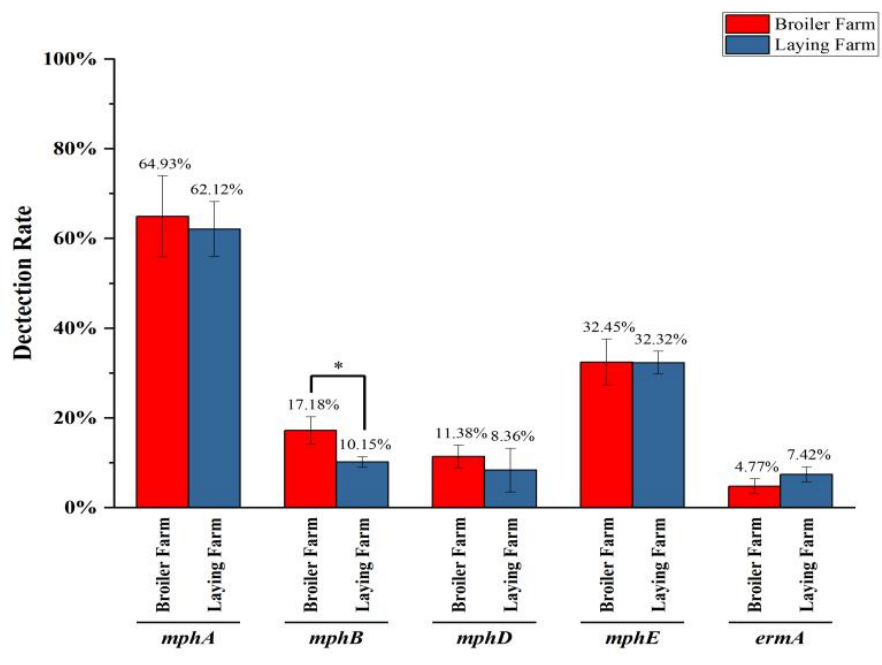

$* p<=0.05 \quad * * p<=0.01 \quad * * * p<=0.001$

597

598

D

599

600

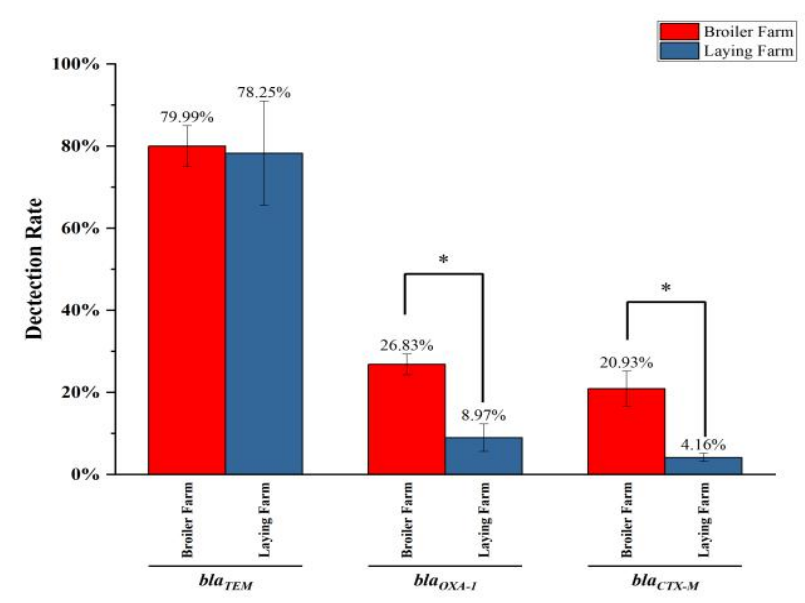


$\mathbf{E}$

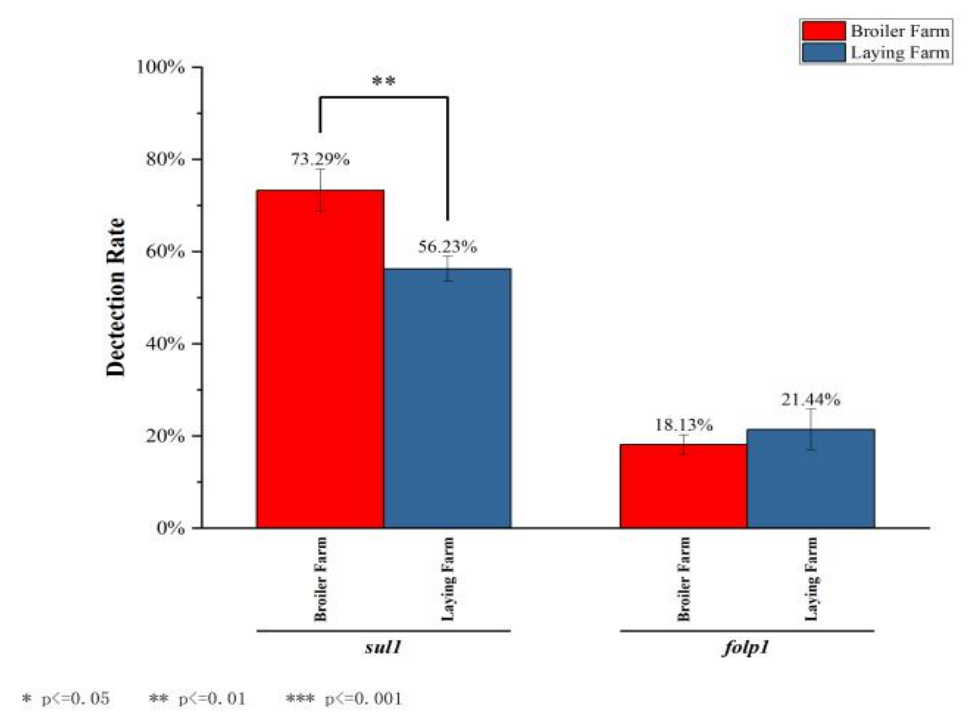

601

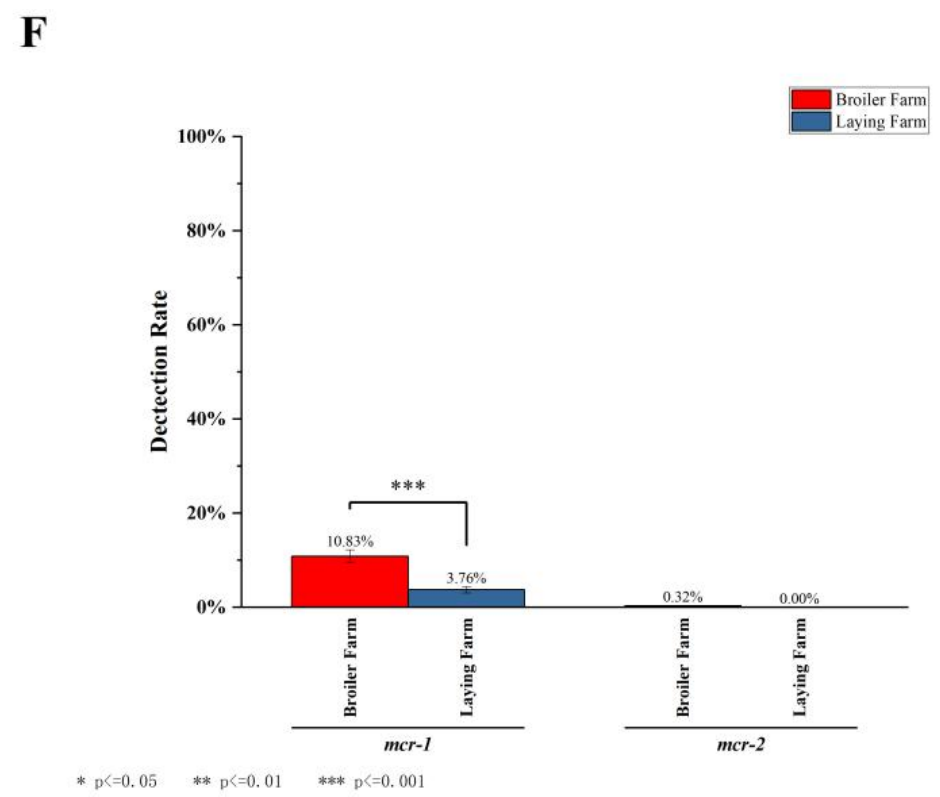

602 Figure 8 The complete sequence circular diagram of p20432-mcr and R478. The 603 innermost ring represents $(\mathrm{G}-\mathrm{C}) /(\mathrm{G}+\mathrm{C})$; the blue ring indicates $\mathrm{GC}$ content, and the 604 outward depression indicates that the GC content is higher than the mean value. Black 605 area in the outer circle represents the backbone area and gray area represents the 606 accessory modules. The outermost circle shows the distribution of genes represented 607 by colored arrows in the plasmid. 

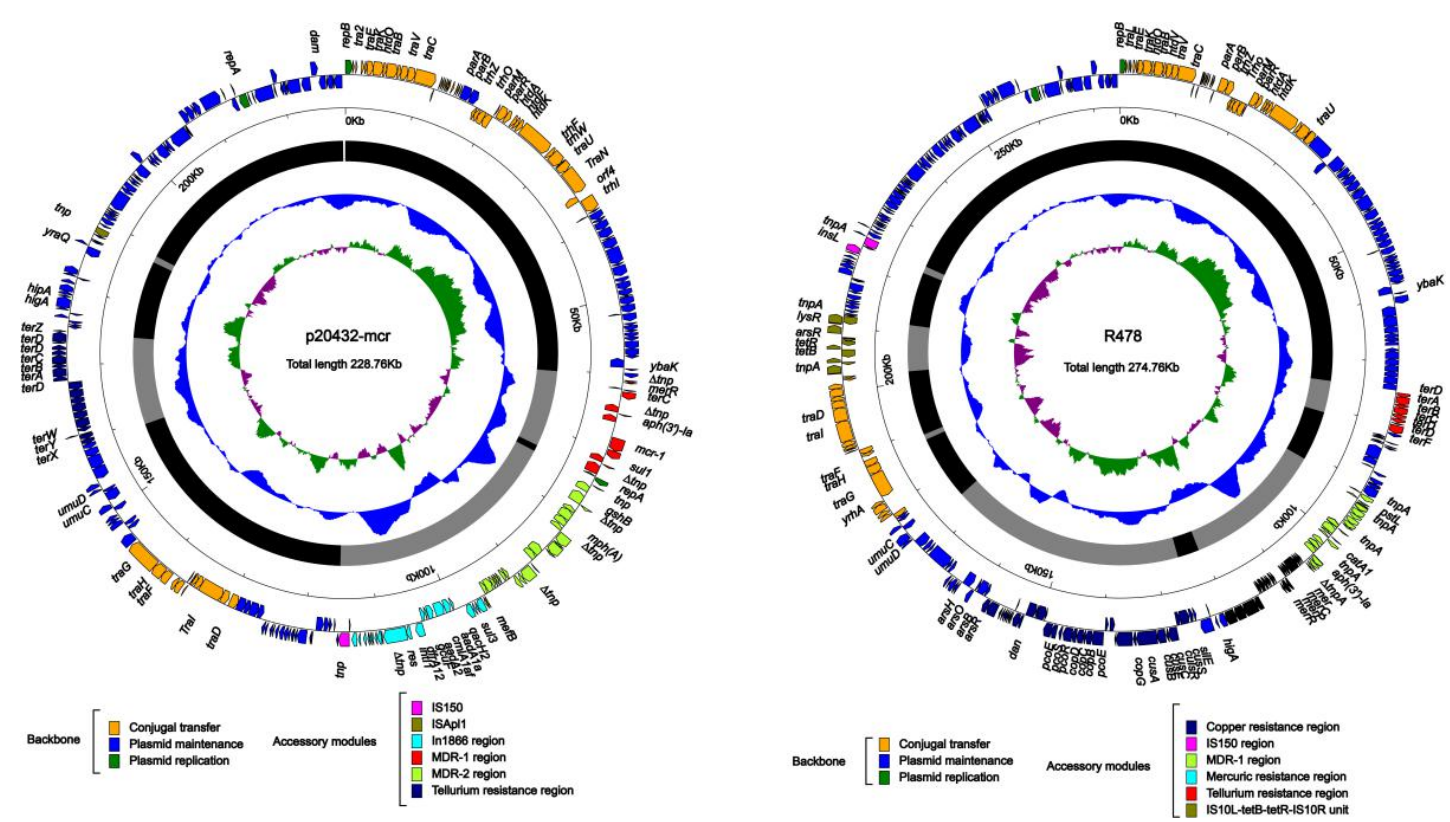

Figure 9 The complete sequence circular diagram of p2021-mcr. (A) The innermost ring represents $(\mathrm{G}-\mathrm{C}) /(\mathrm{G}+\mathrm{C})$; the blue ring indicates $\mathrm{GC}$ content, and the outward depression indicates that the GC content is higher than the mean value. Black area in

613 the outer circle represents the backbone area and gray area represents the accessory

614 modules. The outermost circle shows the distribution of genes represented by colored

615 arrows in the plasmid. (B) Linear comparison of p2021-mcr and pAH62-1. Shadows

616 indicate nucleotide homology is greater than $95 \%$. (C) The diversity of $m c r-1$

617 upstream environment of $r c i$ in Inc2 plasmid.

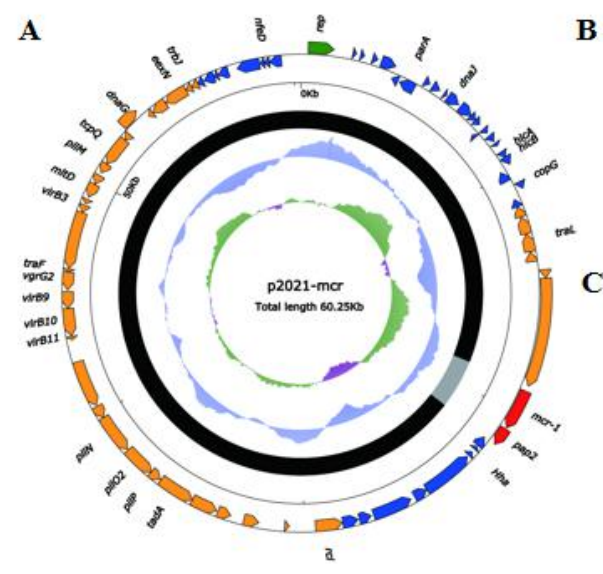

B
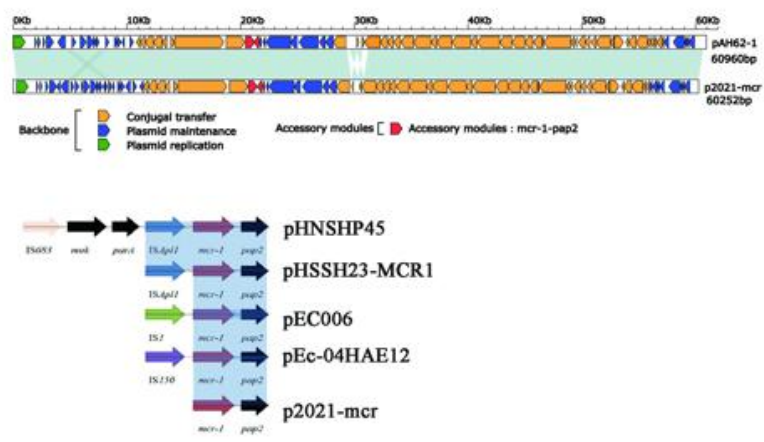

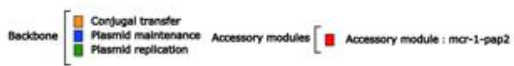


618 Figure 10 The organization and alignment of MDR-1 region of p20432-mcr. (A) 619 Different colors indicate different moving elements, and arrows indicate genes. 620 Shading indicates that the homology of the two parts of nucleic acids is greater than $62195 \%$, and the number in parentheses indicates the location of the fragment in the 622 plasmid. (B) The organization and alignment of MDR-2 region and In1866-related 623 region of p20432-mcr. Different colors indicate different moving elements, and 624 arrows indicate genes. Shading indicates that the homology of the two parts of nucleic 625 acids is greater than $95 \%$, and the number in parentheses indicates the organization 626 and alignment of MDR-2 region and In 1866-related region of p20432-mcr.

627

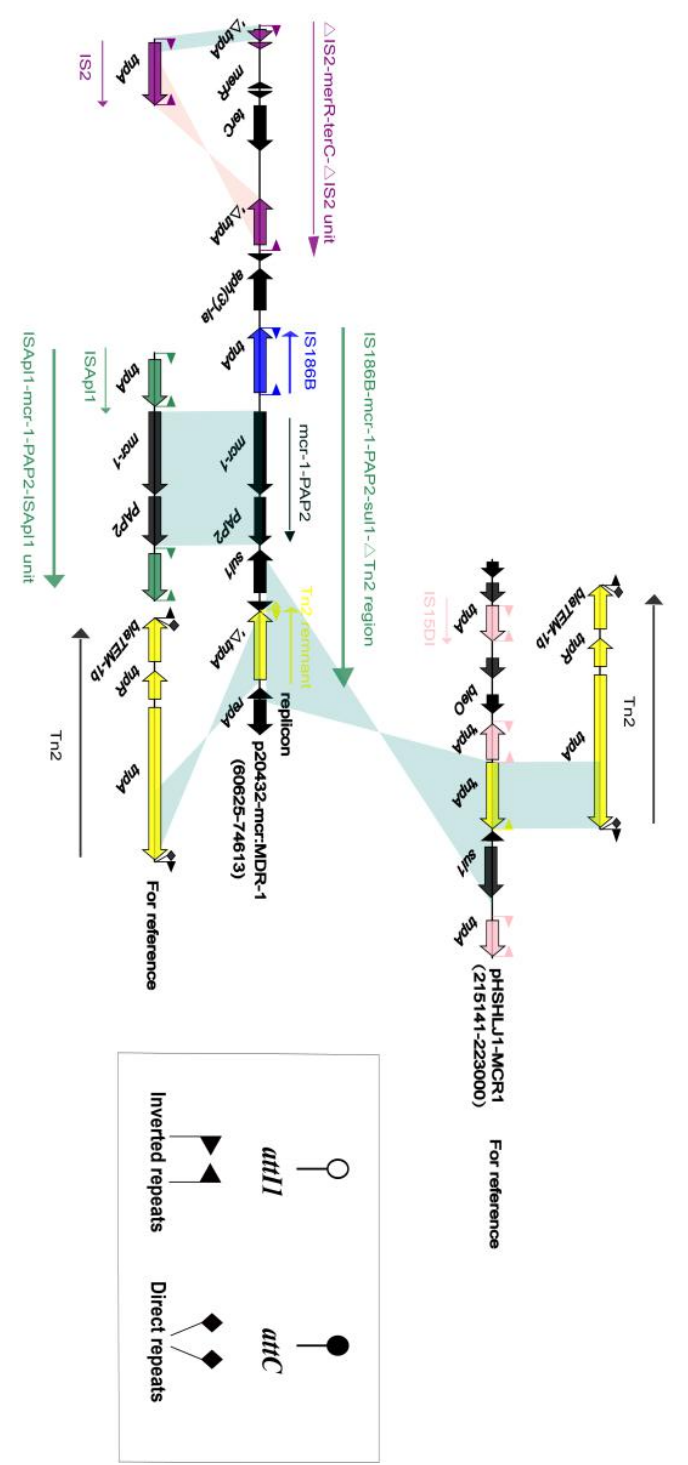




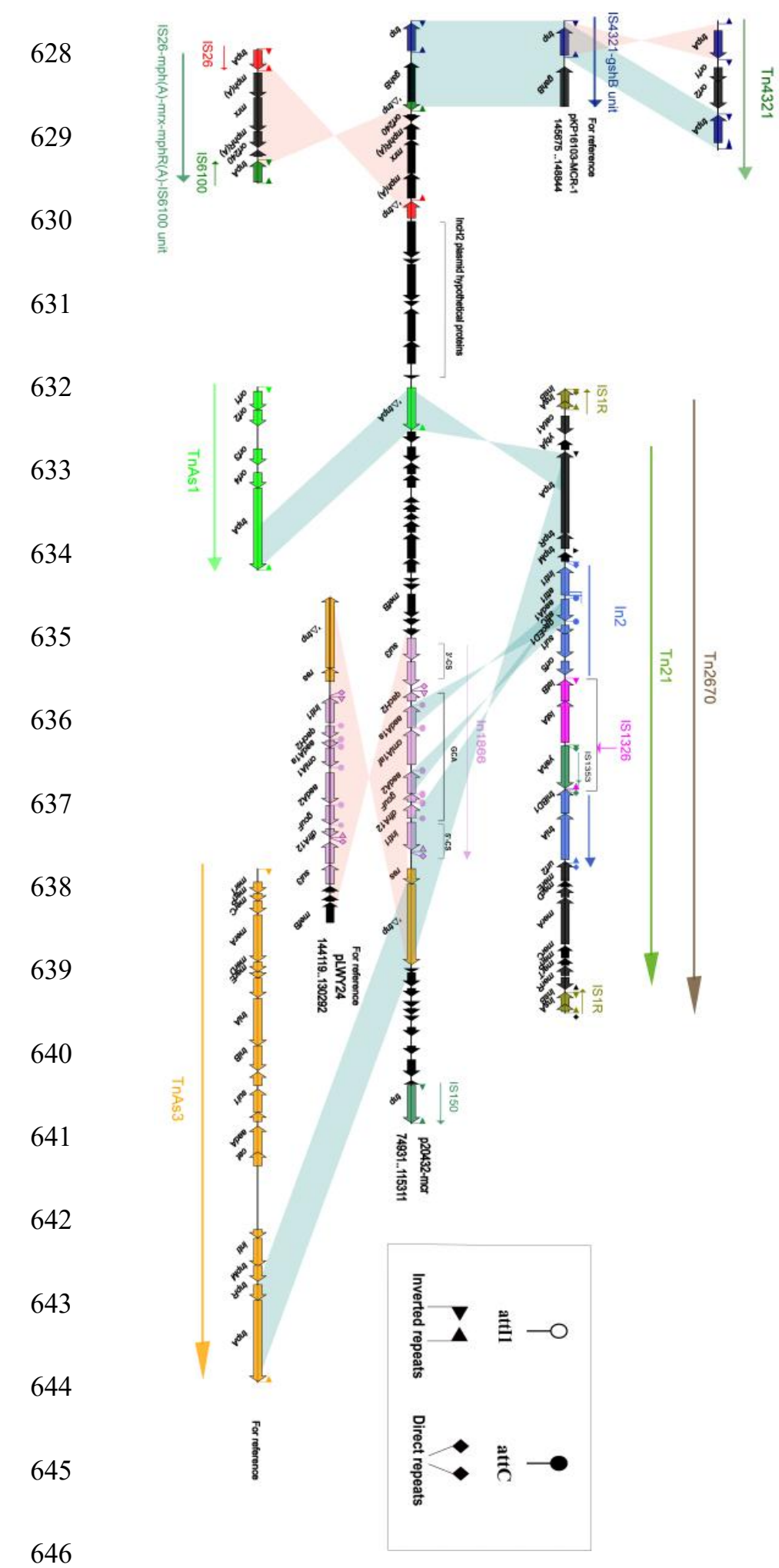

647

648

649 
651 Table1 The result of MIC by using VITEK-2

652

\begin{tabular}{|c|c|c|c|c|c|c|}
\hline \multirow[t]{2}{*}{ Category } & \multirow[t]{2}{*}{ Antimicrobial agent } & \multirow[b]{2}{*}{ EC600 } & \multirow[b]{2}{*}{ RB20432 } & \multicolumn{3}{|c|}{ MIC (mg/L)/antimicrobial susceptibility } \\
\hline & & & & $\begin{array}{c}\text { RB20432-mcr-1 } \\
\text {-EC600 }\end{array}$ & DB20D28 & $\begin{array}{c}\text { DB20D28-mcr-1- } \\
\text { EC600 } \\
\end{array}$ \\
\hline \multirow[t]{2}{*}{ Penicillin } & Ampicillin & $8 / \mathrm{S}$ & $\geq 256 / \mathrm{R}$ & $\geq 256 / \mathrm{R}$ & $\geq 256 / \mathrm{R}$ & $\geq 256 / \mathrm{R}$ \\
\hline & Amoxicillin & $\leq 4 / \mathrm{S}$ & $\geq 256 / \mathrm{R}$ & $\geq 256 / \mathrm{R}$ & $\geq 256 / \mathrm{R}$ & $\geq 256 / \mathrm{R}$ \\
\hline \multirow[t]{2}{*}{$\beta$-lactam } & Ceftazidime & $\leq 4 / \mathrm{S}$ & $\leq 4 / \mathrm{S}$ & $\leq 4 / \mathrm{S}$ & $\leq 4 / \mathrm{S}$ & $\leq 4 / \mathrm{S}$ \\
\hline & Imipenem & $\leq 1 / \mathrm{S}$ & $\geq 16 / \mathrm{R}$ & $\leq 1 / \mathrm{S}$ & $\leq 1 / \mathrm{S}$ & $\leq 1 / \mathrm{S}$ \\
\hline Colistin & Polymyxin B & $\begin{array}{c}\leq 0.5 / \\
\mathrm{S}\end{array}$ & $\geq 8 / \mathrm{R}$ & $\geq 8 / \mathrm{R}$ & $4 / \mathrm{I}$ & $\geq 8 / \mathrm{R}$ \\
\hline Tetracycline & Tetracycline & $4 / \mathrm{S}$ & $\geq 128 / \mathrm{R}$ & $\geq 128 / \mathrm{R}$ & $\geq 128 / \mathrm{R}$ & $\geq 128 / \mathrm{R}$ \\
\hline \multirow[t]{3}{*}{ Aminoglycosides } & kanamycin & $8 / \mathrm{S}$ & $\geq 128 / \mathrm{R}$ & $32 / \mathrm{I}$ & $\geq 64 / \mathrm{R}$ & $\geq 64 / \mathrm{R}$ \\
\hline & Azithromycin & $8 / \mathrm{S}$ & $\geq 128 / \mathrm{R}$ & $\geq 64 / \mathrm{R}$ & $\leq 4 / \mathrm{S}$ & $\leq 4 / \mathrm{S}$ \\
\hline & Gentamicin & $\leq 1 / \mathrm{S}$ & $\geq 16 / \mathrm{R}$ & 8/I & $\leq 1 / \mathrm{S}$ & $\leq 1 / \mathrm{S}$ \\
\hline Quinolines & Ciprofloxacin & $\leq 0.25 / \mathrm{S}$ & $\geq 4 / \mathrm{R}$ & $\geq 4 / \mathrm{R}$ & $\leq 0.25 / \mathrm{S}$ & $\leq 0.25 / \mathrm{S}$ \\
\hline Sulfonamides & Sulfamethoxazole & $\leq 20 / \mathrm{S}$ & $\geq 320 / \mathrm{R}$ & $\geq 320 / \mathrm{R}$ & $\geq 320 / \mathrm{R}$ & $\geq 320 / \mathrm{R}$ \\
\hline
\end{tabular}

S, Susceptible; R, Resistant; I, Intermediate.

653

654 


\section{Supplementary Files}

This is a list of supplementary files associated with this preprint. Click to download.

- TableS1.docx

- Tables2.docx

- supplementarydata.xlsx

- Figures1.docx 